Canad. Math. Bull. Vol. 21 (3), 1978

\title{
A NOTE ON THE LENGTH OF TRIVECTORS
}

\author{
J. A. MACDOUGALL
}

This note concerns elements (called trivectors) of the third Grassmann product of a complex vector space $U$. Usually there are many ways to write a given trivector as the sum of simple or decomposable trivectors, and it is an interesting problem to find those representations which contain the smallest possible number of decomposables. This number we shall call the length of the trivector. Let $N(n)$ denote the length of the longest trivector in $\wedge^{3} U$ where $U$ has dimension $n$. In this note we give upper bounds for $N(n)$ when $n \leq 8$.

When $\operatorname{dim} U=4$, it is well known that $\bigwedge^{3} U$ consists entirely of decomposables, so that the length of any non-zero trivector is 1 . If $\operatorname{dim} U=5$, we make use of the length-preserving isomorphism between $\wedge^{3} U$ and $\bigwedge^{2} U$. It is well known that the maximum length of an element in $\wedge^{2} U$ is [n/2], which gives $N(5)=2$. Capdevielle [1] has shown that $N(6)=3$.

For $n=7$ and 8 , Gurevich $[2,3]$ has determined canonical forms into which all trivectors may be put, showing that $N(7) \leq 5$ and $N(8) \leq 7$. However, some of his expressions may be shortened to yield the following bounds: $N(7) \leq 4$, $N(8) \leq 5$. Let $a, b, c \cdots$ be vectors of $U$ and denote exterior products such as $a \wedge b \wedge c$ simply by $a b c$. For $u=7$, the only form with more than 4 decomposables may be shortened as follows:

$$
a q p+b r p+c s p+q r s+a c b=(a-r)(q+b) p+q r(s-p)-a b(c+p)+c s p
$$

showing that $N(7) \leq 4$.

For $n=8$, all forms with more than 5 decomposables may be shortened as follows:

(I) $a b c+q r s+a q p+b s p+c r p+b r t+c s t$

$$
=(b+q+c)(r+s)(p+s)+(a+r+s) q p+(t-p-s) b r+(r+t-p) c s+a b c
$$

(II) $a b c+q r s+a q p+b s p+b r t+c s t$

$$
=(r-c) t(b+s)+c(a+t) b+(q+t) r s+a q p+b s p
$$

(IV) $q r s+a q p+b s p+c r p+b r t+c s t$

$$
=p(b+c)(r+s)+(t-p) c s+(t-p) b r+q r s+p a q
$$

Received by the editors August 29, 1977. 
(IX) $a b c+q r s+a q p+b s p+c r p+c s t$

$$
=(b-r)(c+s) p+(q-p) r s+(a+b)(b+p) c+c s t+a(c+q) p
$$

Thus proves that $N(8) \leq 5$.

REMARKs: (1) There doesn't appear to be any reason to expect $N(n)$ to be a linear function and it may be that $N(8)=4$.

(2) Although Gurevich does not give proofs for his results, it seems likely that he restricts himself to $C$ for the algebraic closure so that the above bounds may be true for any algebraically closed field.

\section{REFERENCES}

1. Bernadette Capdevielle, Classifications des Formes Trilinéaires Alternées en Dimension 6, Enseign. Math., t. 18, 1972-73, pp. 225-243.

2. G. B. Gurevich, Sur Les Trivecteurs Dans l'Espace à Sept Dimensions, Dok. Akad. Nauk SSSR III, No. 8-9, 1934, pp. 567-569.

3. G. B. Gurevich, Classification des Trivecteurs Ayant le Rang Huit, Dok. Akad. Nauk SSSR II, No. 5-6, 1935, pp. 355-356.

Department of Mathematics

UNIVERSITY OF PRINCE EdWARD IsLAND

Charlottetown, Prince Edward Island 